\title{
INCORPORAÇÃO DE RESÍDUO DE MADEIRA EM LADRILHO HIDRÁULICO VIBRADO
}

Alessandra Savazzini-Reis ' Alana Franzin Fagundes ${ }^{2}$

\section{Resumo}

A indústria moveleira brasileira se caracteriza pelo predomínio de pequenas e medias empresas, sendo que os móveis de madeira representam cerca de $80 \%$ do mercado total. A região de Colatina-ES faz parte de um grande pólo moveleiro do país, o qual gera resíduos de madeira provenientes das marcenarias. Neste contexto, foi proposta a incorporação do resíduo de madeira em ladrilho hidráulico vibrado. O resíduo de madeira substituiu a areia nos teores de $5 \%$ e $10 \%$ (massa) na argamassa cimenticia do ladrilho. Os ladrilhos foram moldados por vibração e submetidos a análises dimensional e geométrica, avaliação da resistência à flexão e da absorção de água. Os resultados mostraram ser possível incorporar $5 \%$ de resíduo de madeira na confecção do ladrilho hidráulico vibrado e manter as propriedades mecânicas quando comparado ao ladrilho de referência.

Palavras-chave: Resíduo de madeira; Ladrilho hidráulico vibrado; Resíduo de marcenaria; Cimento-madeira.

\section{INCORPORATION OF WOOD WASTE IN VIBRATED HYDRAULIC TILE}

\begin{abstract}
The Brazilian furniture industry is characterized by the predominance of small and medium-sized companies, with wooden furniture accounting for about $80 \%$ of the total market. The region of Colatina-ES is part of a large furniture pole of the country, which generates wood wastes coming from the joinery. In this context, it was proposed to incorporate the wood waste in vibrated hydraulic tile. The wood waste replaced the sand at $5 \%$ and $10 \%$ (mass) in the cement mortar of the tile. The tiles were molded by vibration and subjected to dimensional and geometric analysis, evaluation of the flexural strength and water absorption. The results showed that it is possible to incorporate $5 \%$ of wood waste in the production of the vibrated hydraulic tile and to maintain the mechanical properties when compared to the reference tile. Keywords: Wood waste; Vibrated hydraulic tile; Joinery waste; Cement-wood.
\end{abstract}

\section{INTRODUÇÃO}

A técnica e a arte de trabalhar a madeira tem evoluído ao longo do tempo surgindo várias aplicações, e com isso foram geradas grandes áreas de desmatamentos [I]. $\mathrm{O}$ aumento das restrições ambientais tem resultado no uso de madeiras reflorestáveis, tais como, pinos, eucalipto, além de chapas e painéis de madeira reconstituída (medium density fiberboard-MDF) em substituição às madeiras nativas. Os móveis de madeira representam cerca de $80 \%$ do mercado total da indústria moveleira do Brasil, o qual apresentou uma produção de 430,6 milhões de peças em 2016 ocupando ○ $5^{\circ}$ lugar como produtor mundial [2]. Nesse mesmo ano, o setor de árvores plantadas brasileiro gerou 47,8 milhões de toneladas de resíduos sólidos, apresentando assim um aumento em relação a estimativa de geração anual do resíduo de madeira no Brasil de 30 milhões de toneladas [3], sendo $91 \%$ do resíduo proveniente da indústria madeireira, $6 \%$ da construção civil e $3 \%$ do meio urbano (poda de árvore). A região da pesquisa, localizada em Colatina-ES faz parte de um grande polo moveleiro do país, o qual gera grande volume de resíduos de madeira provenientes das marcenarias. Apesar das estimativas de geração de resíduos, o setor madeireiro brasileiro não tem um controle eficaz de quantificação de volume fabricado de peças e de resíduos gerados e grande parte do resíduo de madeira é descartado a céu aberto [4] podendo acarretar contaminação ambiental [5].

O resíduo em questão não é comercialmente usado em matrizes cimentícias, apenas uma parcela dele é usado como combustível em fornos de olarias existentes em

'Coordenadoria de Edificações, Instituto Federal do Espírito Santo - IFES, Campus Vitória, Vitória, ES, Brasil. E-mail: alessandrar@ifes.edu.br ${ }^{2}$ Coordenadoria de Arquitetura e Urbanismo, Instituto Federal do Espírito Santo - IFES, Campus Colatina, Colatina, ES, Brasil. Email: alanafranzin@hotmail.com 
Colatina-ES. Quanto aos resíduos orgânicos em geral não foi encontrada utilização em escala comercial em matrizes cimentícias.

Já o ladrilho hidráulico é uma placa cimentícia paralelepipédica usada em revestimento de piso interno e externo, sendo composto por cimento Portland, agregados, água, aditivos e pigmentos [6]. O ladrilho hidráulico tem sido usado como piso tátil em calçadas que atendem a NBR 9050/20I5 [7] provendo acessibilidade; além de ser usado como elemento decorativo na arquitetura.

Neste contexto, foi proposta a incorporação do resíduo de madeira proveniente de marcenaria como matéria prima do ladrilho hidráulico vibrado.

\section{I.I Classificação do Resíduo de Marcenaria}

O resíduo de madeira pode ser dividido de acordo com sua morfologia em: cavaco (dimensões máximas de $50 \times 20 \mathrm{~mm}$ ), maravalha (dimensões maiores que 2,5 mm), serragem (dimensões entre 0,5 e 2,5 mm) e pó de serra (dimensões menores que $0,5 \mathrm{~mm}$ ) [8]. Segundo a NBR I0004/2004 [9] resíduo de madeira é classificado como sendo resíduo "não perigoso" constando no Anexo H com código de identificação A009.

\section{I.2 Utilização do Resíduo de Madeira em Matrizes Cimenticias}

Pesquisas com o resíduo de madeira em argamassa cimenticia substituindo a areia mostram que resistência mecânica e massa específica diminuem enquanto a absorção de água e índice de vazios aumentam com o aumento do teor de resíduo, alcançando teor máximo de 10\% de resíduo. Em geral, é feito uso de aditivo acelerador de pega ou plastificante para diminuir efeitos negativos entre cimento Portland e o resíduo de madeira, como o aumento do tempo de pega $[10,11]$. Em testes do resíduo de madeira em argamassa de cimento e areia $I: I$ a $I: 3$ substituindo a areia em teores $2 \%$ a $5 \%$ (massa), o valor da resistência à compressão mais próximo da referência foi obtida no traço I:3 com $5 \%$ de resíduo $[12,13]$.

No concreto de cimento Portland, o resíduo também ocasiona perda na resistência mecânica [14], em contrapartida, o resíduo gera boa retenção de ruído e energia térmica $[15,16]$.
Já a granulometria do resíduo pode influenciar a densidade e a resistência mecânica da argamassa, o intervalo de tamanho de partículas de $1,7 \mathrm{~mm}$ a 0,85 $\mathrm{mm}$ comparado ao de $0,50 \mathrm{~mm}$ a $0,25 \mathrm{~mm}$, apresentou melhores resultados no compósito cimento-madeira [17]. O pó de madeira substituiu a areia em argamassas nos traços 2: I e 3:I (massa), a absorção de água, massa específica e capilaridade de blocos foram similares aos valores de referência [4]. Foi testado também o resíduo de madeira com lodo de estação de tratamento de água para serem usadas em concreto leve [18].

\section{MATERIAIS E MÉTODOS}

\section{I Resíduo de madeira}

O resíduo de madeira, coletado em marcenaria situada em Colatina-ES, foi passado na peneira de mesh 4 (abertura de malha de 4,8 $\mathrm{mm}$ ) que corresponde a granulometria da areia [19], de forma que o resíduo é composto por maravalha, serragem e pó de serra. Com base em tratamentos feitos no resíduo de madeira $[4,14,20]$ para diminuir a influência no cimento Portland, devido a madeira possuir componentes como hemicelulose, açúcares, taninos e lignina que retardam a cura e a resistência mecânica do compósito cimento-madeira [2I], foi feito tratamento do resíduo por imersão em solução de cal hidratada por Ih com um traço 4,7:I (cal:água) sem lavagem e secagem em mufla a $105^{\circ} \mathrm{C}$ por $24 \mathrm{~h}$.

\subsection{Caracterização da Areia e do Cimento Portland}

Os procedimentos dos ensaios de caracterização da areia e do cimento Portland CPV-ARI seguiram as Normas da $A B N T$ e os resultados mostram que os materiais podem ser usados na argamassa cimentícia do ladrilho hidráulico vibrado, pois atendem aos limites especificados nas Normas da ABNT para utilização em argamassa e concreto (Tabela I). A areia se apresenta bem graduada e quartzoza, com módulo de finura se situando na faixa da zona ótima entre 2,20 e 2,90 para utilização em concreto. E o cimento atende também aos valores indicados pelo fabricante "Liz Cimentos" para a classe do cimento CPV-ARI estando apto a ser usado em produtos cimenticios.

Tabela I. Ensaios de caracterização da areia e cimento CP-V-ARI

\begin{tabular}{cccc}
\hline Material & Ensaio & Norma & Resultado \\
\hline Areia & Granulometria & NBR NM 248/2003 [19] & MF $=2,64 \mathrm{DMC}=2,36 \mathrm{~mm}$ \\
& Massa específica & NBR 9776/1987 [22] & $2,63 \mathrm{~g} / \mathrm{cm}^{3}$ \\
& Massa unitária & NBR NM 45/2006 [23] & $1,57 \mathrm{~g} / \mathrm{cm}^{3}$ \\
Cimento Portland & Finura \#200 & NBR II I579/2012 [24] & $2,2 \%$ \\
CPV-ARI & Massa específica & NBR I6605/2017 [25] & $2,96 \mathrm{~g} / \mathrm{cm}^{3}$ \\
& Consistência normal & NBR I6606/2017 [26] & $31 \%$ \\
& Tempos de pega & NBR I6607/20I7 [27] & $2: 35 \mathrm{~h}-3: 35 \mathrm{~h}$ \\
\hline
\end{tabular}

Nota: $M F=$ módulo de finura; $\mathrm{DMC}=$ dimensão máxima característica. 


\subsection{Moldagem do Ladrilho Hidráulico Vibrado}

O molde usado é feito de plástico com dimensões de $200 \mathrm{~mm} \times 200 \mathrm{~mm}$ e espessura de $15 \mathrm{~mm}$. Foi feito um teste de moldagem do ladrilho hidráulico tendo como base os dados de dosagem I:2 (cimento:areia) de Reis e Tristão [28] usados em ladrilho hidráulico prensado. A partir daí, o resíduo de madeira substituiu a areia em teores (massa) variando entre $5 \%$ e $25 \%$ (Tabela 2 ).

Os materiais foram misturados em argamassadeira e lançados no molde plástico, que foi vibrado em vibrador de peneiras marca Contenco $\mathrm{Cl} 016$ por 80 segundos a $45 \mathrm{~Hz}$. Foi feita cura ao ar por 24h, desforma e imersão em água. Foi verificado que com o aumento do teor de resíduo de madeira (Figura la), o ladrilho demandou mais água para apresentar consistência ideal para vibração e com isso sua secagem se prolongou por mais de $48 \mathrm{~h}$ com $5 \%$ de resíduo, e com $15 \%$ e $25 \%$ de resíduo os ladrilhos quebraram na desforma (Figuras Ib, Ic).

Tabela 2. Dosagem do ladrilho hidráulico $(5 \%$ a $25 \%$ de resíduo de madeira)

\begin{tabular}{cccccc}
\hline \multirow{4}{*}{ Traços } & Teor resíduo & cimento & areia & resíduo & água \\
\cline { 2 - 6 } (massa) & $5 \%$ & $\mathrm{I}$ & $\mathrm{I}, 9$ & $0, \mathrm{I}$ & 0,60 \\
& $15 \%$ & $\mathrm{I}$ & $\mathrm{I}, 7$ & 0,3 & $\mathrm{I}, 10$ \\
& $25 \%$ & $\mathrm{I}$ & $\mathrm{I}, 5$ & 0,5 & $\mathrm{I}, 60$ \\
\hline
\end{tabular}

Tabela 3. Dosagem do ladrilho hidráulico (5\% a I5\% de resíduo de madeira)

\begin{tabular}{cccccc}
\hline & Teor resíduo & cimento & areia & resíduo & água \\
\cline { 2 - 6 } Traços & $0 \%$ & $\mathrm{I}$ & 2,0 & 0 & 0,50 \\
(massa) & $5 \%$ & $\mathrm{I}$ & $\mathrm{I}, 9$ & 0,1 & 0,50 \\
& $10 \%$ & $\mathrm{I}$ & $\mathrm{I}, 8$ & 0,2 & 0,65 \\
& $\mathrm{I} \%$ & $\mathrm{I}$ & $\mathrm{I}, 7$ & 0,3 & 0,80 \\
\hline
\end{tabular}

Tabela 4. Avaliação dimensional e geométrica do ladrilho hidráulico

\begin{tabular}{ccc}
\hline Propriedade & Limite & Tolerância \\
\hline Comprimento & máximo $400 \mathrm{~mm}$ & $\pm 3 \mathrm{~mm}$ \\
Largura & mínimo $100 \mathrm{~mm}$ & $\pm 3 \mathrm{~mm}$ \\
Espessura & mínimo $18 \mathrm{~mm}$ & $\pm 1 \mathrm{~mm}$ \\
Esconsidade & máx. $3 \mathrm{~mm}$ & - \\
Empenamento & máx. $3 \mathrm{~mm}$ & - \\
Ortogonalidade & não pode ser perceptível a olho nú \\
\hline
\end{tabular}

Com a dificuldade de se obter a consistência ideal para uma vibração eficiente foi adicionado aditivo plastificante Cemix (marca Otto Baumgart), na proporção de 0,2\% da massa de cimento Portland.

Foram confeccionados ladrilhos com teores de resíduo reduzidos para $5 \%, 10 \%$ e I $5 \%$, relação água cimento inicial de 0,50 e $1,5 \mathrm{ml}$ de aditivo plastificante além de ladrilhos de referência com $0 \%$ de resíduo (Tabela 3 ).

A desforma ocorreu com $24 \mathrm{~h}$ para os ladrilhos feitos com $0 \%, 5 \%$ e $10 \%$ de resíduo, porém com $15 \%$ ainda houve aumento da relação a/c para vibração adequada, apesar do tratamento com hidróxido de cálcio prévio realizado e a desforma ocorreu com quebras em mais de 24h. Assim, foram definidos os teores de resíduo de $0 \%, 5 \%$ e $10 \%$, já descritos na Tabela 3, para confecção dos ladrilhos.

Não foram executados ensaios de envelhecimento com os materiais utilizados na produção do ladrilho hidráulico.

\subsection{Avaliação Dimensional e Geométrica do Ladrilho Hidráulico Vibrado}

As propriedades avaliadas e os limites seguiram a NBR 9457/2013 [6] (Tabela 4). Para o lote amostral ser aceito nenhuma das 9 peças testadas pode ser rejeitada. A Figura 2 apresenta detalhes dos ensaios.

\subsection{Avaliação da Resistência à Flexão e da Absorção de Água do Ladrilho Hidráulico Vibrado}

Os ensaios seguiram a NBR 13818/1997 [29], o ensaio de flexão foi feito em prensa EMIC DL 30000. Para - lote amostral de 6 peças ser aceito nenhuma das peças pode ser rejeitada. $O$ teste de absorção de água foi feito com objetivo de verificar possível relação inversa com a resistência à flexão.

\section{RESULTADOS E DISCUSSÃO}

\section{I Avaliação Dimensional e Geométrica do Ladrilho Hidráulico Vibrado}

Pode-se observar na Tabela 5 que largura e comprimento atendem ao valor nominal $(200 \mathrm{~mm})$ e tolerância $(3 \mathrm{~mm})$ de Norma. Quanto à espessura, não atende ao valor mínimo

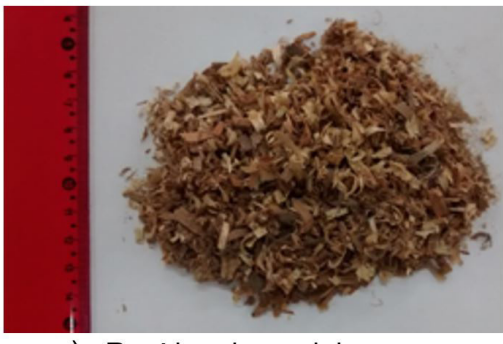

a) Resíduo de madeira

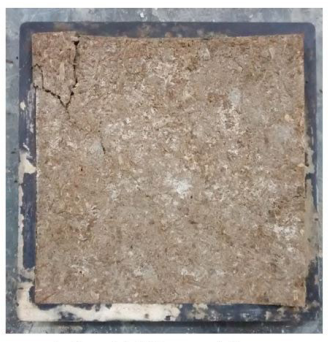

b) $15 \%$ resíduo

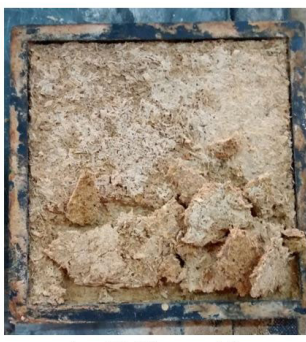

c) $25 \%$ resíduo

Figura I. Detalhes da moldagem do ladrilho. 


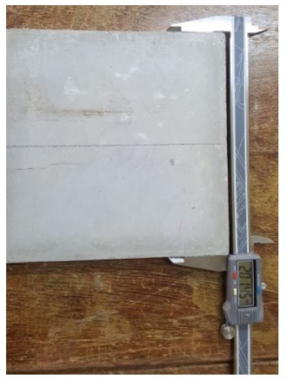

a) Largura

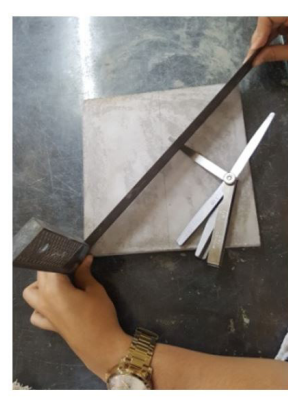

b) Empenamento

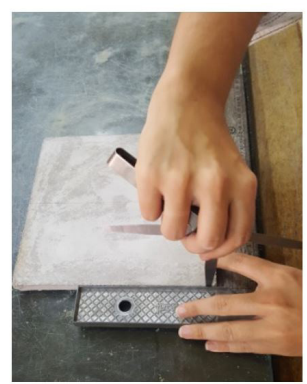

c) Esconsidade

Figura 2. Detalhes da avaliação dimensional e geométrica.

Tabela 5. Resultados da avaliação dimensional e geométrica do ladrilho hidráulico

\begin{tabular}{cccccc}
\hline \multirow{2}{*}{$\begin{array}{c}\text { Teor resíduo } \\
(\%)\end{array}$} & Largura & Comprimento & Espessura & Empenamento & Esconsidade \\
\cline { 2 - 6 } & 201,50 & 201,52 & 13,15 & 0 a 0,30 & 0 a 0,90 \\
5 & 201,53 & 201,63 & 13,72 & 0,05 a 0,25 & 0 a 0,25 \\
10 & 201,56 & 201,49 & 13,47 & 0 a 0,40 & 0,35 a 0,45 \\
\hline
\end{tabular}

Tabela 6. Resultados da resistência à flexão e da absorção de água no ladrilho hidráulico

\begin{tabular}{ccc}
\hline $\begin{array}{c}\text { Teor resíduo } \\
(\%)\end{array}$ & $\begin{array}{c}\text { Resistência à flexão } \\
(\mathbf{M P a})\end{array}$ & $\begin{array}{c}\text { Absorção de } \\
\text { água (\%) }\end{array}$ \\
\hline 0 & $5,91 \pm 1,23$ & $10,07 \pm 0,30$ \\
5 & $3,71 \pm 0,74$ & $16,14 \pm 2,25$ \\
10 & $3,01 \pm 0,82$ & $18,74 \pm 2,18$ \\
\hline
\end{tabular}

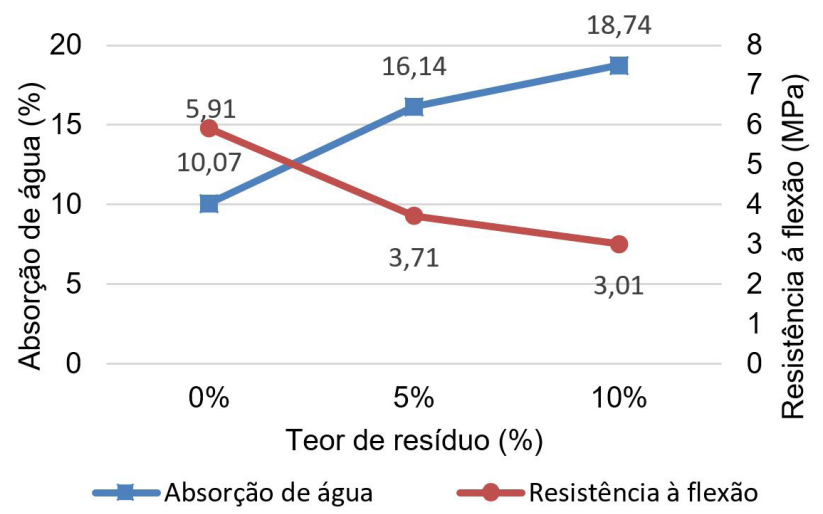

Figura 3. Propriedades física e mecânica x teor de resíduo de madeira.

de $18 \mathrm{~mm}$ para uso do ladrilho em pavimentação. O limite máximo de $1 \mathrm{~mm}$ para empenamento e esconsidade foi obedecido por todos ladrilhos, além do atendimento à ortogonalidade verificada visualmente.

\subsection{Resistência à Flexão e Absorção de Água do Ladrilho Hidráulico Vibrado}

As médias e desvios padrão da absorção de água e da resistência à compressão medidos nos corpos de prova são apresentados na Tabela 6. Observa-se que o maior valor de resistência à flexão e o menor valor de absorção de água foram obtidos no teor de $5 \%$ de resíduo. Reafirma-se com isso, a relação indiretamente proporcional que existe entre essas duas propriedades quando medidas em materiais cimentícios.

Pesquisas já realizadas $[12,13]$ encontraram teores do resíduo de madeira variando de 3 a $5 \%$, o que corrobora o teor de $5 \%$ (massa) deste trabalho, com o qual o ladrilho hidráulico atendeu ao valor de resistência à flexão de 3,5 MPa estipulado na Norma [6] e apresentou o menor valor de absorção de água.

Com a adição do resíduo que apresentava granulometria com poucos finos, os poros vazios existentes entre os grãos na mistura não foram preenchidos pelo resíduo diminuindo a compacidade, o que ocasiona o aumento na absorção de água levando à diminuição da resistência mecânica [30].

A moldagem dos ladrilhos hidráulicos por meio de vibração, que ocorreu na pesquisa, também pode ocasionar maior incidência de vazios quando comparados à moldagem por meio de prensagem, o que resulta em baixa resistência mecânica [14]. Por outro lado, um alto índice de vazios resulta em peças mais leves, e esses vazios podem contribuir no desempenho térmico do material [11-16].

$\mathrm{Na}$ Figura 3 que apresenta o comportamento da absorção de água e da resistência à flexão dos ladrilhos observa-se a relação inversamente proporcional entre as duas propriedades corroborando os resultados de outros autores [12-|4].

\section{CONCLUSÃO}

O estudo do teor de resíduo de madeira no ladrilho hidráulico, de $5 \%$ a $10 \%$ (massa), confirmou que a incorporação do resíduo aumenta o tempo de pega do cimento Portland e demanda mais água para moldagem por meio de vibração. 
Já, a adição do aditivo plastificante se mostrou benéfico para atenuação da demanda de água na moldagem.

Os valores das análises dimensional e geométrica nos ladrilhos obedeceram aos limites da norma, apenas a espessura não atendeu ao mínimo estipulado de $18 \mathrm{~mm}$, o que pode ser contornado com moldes que obedeçam a esse requisito.

Os resultados dos ensaios realizados mostram que com o aumento do teor de resíduo de madeira ocorre o aumento da absorção de água e a diminuição da resistência mecânica dos ladrilhos hidráulicos vibrados.

O ladrilho com teor de $5 \%$ atendeu a resistência à flexão de norma, com isso, pode-se dizer que existe a viabilidade técnica da incorporação do resíduo de madeira na fabricação de ladrilho hidráulico vibrado, que pode vir a contribuir para a sustentabilidade dos setores envolvidos.

\section{REFERÊNCIAS}

I Leão MS, Naveiro RM. Móveis: indústria de moveis mostra competitividade de madeira brasileira. Goiânia: CGI Imóveis; 2010 [acesso em 10 maio 2018]. Disponível em: http://www.cgimoveis.com.br/economia/ documento.2010-06-08.6642478173

2 Industria Brasileira de Árvores. Relatório 2019. Elaboração: Poyry Consultoria em Gestão e Negócios Ltda. Brasília: IBÁ; 2019. 80 p.

3 Brasilian Furniture. Dados do setor 2016. 2018. [acesso em 12 maio 2018]. Disponível em: http://www. brazilianfurniture.org.br/sobresetor

4 Arruda PP, Silva AO, Martinez RG. Utilização de resíduos de madeira como elemento construtivo. In: Anais do XIV Encontro Nacional de Tecnologia do Ambiente Construído (ENTAC); 20I2; Juiz de Fora, Brazil. Porto Alegre: ANTAC; 2012.219 p.

5 Argenta DOF. Alternativas de melhoria no processo produtivo do setor moveleiro de Santa Maria/SC: impactos ambientais [dissertação]. Santa Maria: Universidade Federal de Santa Maria; 2007. I 2 I p.

6 Associação Brasileira de Normas Técnicas. NBR 9457. Ladrilhos hidráulicos para pavimentação - Especificação e métodos de ensaio. Rio de Janeiro: ABNT; 20I3. 9 p.

7 Associação Brasileira de Normas Técnicas. NBR 9050. Acessibilidade a edificações, mobiliário, espaços e equipamentos urbanos. Rio de Janeiro: ABNT; 20I5. 148 p.

8 Casilha AC, Podlasek CL, Junior EFC, Silva MC, Mengatto SNF. Indústria moveleira e resíduos sólidos: considerações para o equilíbrio ambiental. Revista Educação \& Tecnologia. 2004;8:209-228.

9 Associação Brasileira de Normas Técnicas. NBR 10004. Resíduos sólidos - Classificação. Rio de Janeiro: ABNT; 2004. $71 \mathrm{p}$.

10 Garcez M, Santos T, Garcez E, Gatto D. Propriedades mecânicas de compósitos cimento-madeira com serragem tratada de Pinus Elliottii. Ciência da Madeira. 20I5;7(I):I6-27.

I I Weber AM, Cechin L, Tokarski RB, Matoski A. Análise da influência do traço nas propriedades do compósito cimento-madeira. Revista Principia. 2017;36:50-59.

I 2 Fonseca F. Contribuição para o desenvolvimento de compósito de cimento-madeira com matérias da região amazônica [dissertação]. Belém: Programa de Pós-graduação em Engenharia Civil, Centro Tecnológico; Universidade Federal do Pará; 2005.

I 3 Macedo NA, Lima AM, Fonseca FO, Lavôr BVA. Analise estatística do comportamento mecânico à compressão do compósito cimento-madeira. Revista Matéria. 201 I; I6(2):658-667.

I4 Garcez M, Santos T, Gatto D. Avaliação das propriedades físicas e mecânicas de concretos pré-moldados com adição de serragem em substituição ao agregado miúdo. Ciência e Engenharia. 2013;22(2):95- 104.

I5 Bijos AP, Zanatta LA. Resíduo de madeira: Caracterização e aplicabilidade junto ao concreto. In: Anais da XIV Jornada Científica das Faculdades Integradas de Cacoal - UNESC; 2016; Criciúma; Brazil. Criciúma: UNESC; 2016. 7 p.

I6 Ribeiro UG. Desempenho térmico, acústico e mecânico de compósitos produzidos com resíduos da indústria madeireira de Porto Velho [trabalho de pós-graduação]. Manaus: Universidade Federal do Amazonas; 2016.

17 Ronquim R. Produção e caracterização físico-mecânica do compósito cimento-madeira com variação granulométrica dos resíduos lignocelulósicos [trabalho de graduação]. Itapeva: Universidade Estadual Paulista Julio de Mesquita Filho; 2013. 
I8 Souza FR, Sales A, Kague CS, Prado JW. Composto à base de lodo de estação de tratamento de água e serragem de madeira para aplicação como agregado graúdo em concreto. In: Anais do XII Encontro Nacional de Tecnologia do Ambiente Construído - ENTAC; 2008; São Paulo, Brazil. Porto Alegre: ANTAC; 2008. Il p.

19 Associação Brasileira de Normas Técnicas. NBR NM 248. Agregados - Determinação da composição granulométrica. Rio de Janeiro: ABNT; 2003. 4 p.

20 Parchen CFA. Compósito madeira cimento de baixo densidade produzidos com compactação vibro dinâmica. [trabalho de pós-graduação]. Manaus: Universidade Federal do Pará; 20I2. I72 p.

2I Rocha JC. Relatório final: aproveitamento dos resíduos de madeira. Florianópolis: Universidade Federal de Santa Catarina; 2004.

22 Associação Brasileira de Normas Técnicas. NBR 9776. Agregados - Determinação da massa específica de agregados miúdos por meio do frasco Chapman - Método de ensaio. Rio de Janeiro: ABNT; 1987. 3 p.

23 Associação Brasileira de Normas Técnicas. NBR NM 45. Agregados - Determinação da massa unitária e do volume de vazios. Rio de Janeiro: ABNT; 2006. 8 p.

24 Associação Brasileira de Normas Técnicas. NBR II579. Cimento Portland - Determinação do índice de finura por meio da peneira $75 \mu \mathrm{m}\left(\mathrm{n}^{\circ} 200\right)$. Rio de Janeiro: ABNT; 2012. $4 \mathrm{p}$.

25 Associação Brasileira de Normas Técnicas. NBR 16605. Cimento Portland e outros materiais em pó Determinação da massa específica. Rio de Janeiro: ABNT, 2017. 4 p.

26 Associação Brasileira de Normas Técnicas. NBR 16606. Cimento Portland — Determinação da pasta de consistência normal. Rio de Janeiro: ABNT; 2017. 8 p.

27 Associação Brasileira de Normas Técnicas. NBR 16607. Cimento Portland — Determinação dos tempos de pega. Rio de Janeiro: ABNT, 2003. 4 p.

28 Reis AS, Tristão FA. Tactile-floor tile hydraulic with addition residue improvement dimension stones. IBRACON Structures and Materials Journal. 2010;3(4):390-419.

29 Associação Brasileira de Normas Técnicas. NBR I38|8. Placas cerâmicas para revestimento - Especificação e métodos de ensaios. Rio de Janeiro: ABNT; 1997. 78 p.

30 Monteiro ACN. Concreto poroso: dosagem e desempenho [Trabalho de conclusão de curso]. Goiânia: Universidade Federal de Goiás; 2010. 36 p.

Recebido em: 28 Nov. 2018

Aceito em: 20 Maio. 2020 